\title{
Multisensor Classification System for Triggering FES in Order to Support Voluntary Swallowing
}

\author{
Benjamin Riebold (1), Holger Nahrstaedt (1), Corinna Schultheiss (2), Rainer O. Seidl (2), \\ Thomas Schauer (1)
}

(1) Control Systems Group, Technische Universität Berlin; Germany. (2) Unfallkrankenhaus, Ear, Nose and Throat Unit, Berlin, Germany

This article is distributed under the terms of the Creative Commons Attribution Noncommercial License (CC BY-NC 4.0) which permits any noncommercial use, distribution, and reproduction in any medium, provided the original author(s) and source are credited.

\begin{abstract}
In dysphagia the ability of elevating the larynx and hyoid is usually impaired. Electromyography (EMG) and Bioimpedance (BI) measurements at the neck can be used to trigger functional electrical stimulation (FES) of swallowing related muscles. Nahrstaedt et al. ${ }^{1}$ introduced an algorithm to trigger the stimulation in phase with the voluntary swallowing to improve the airway closure and elevation speed of the larynx and hyoid. However, due to nonswallow related movements like speaking, chewing or head turning, stimulations might be unintentionally triggered. So far a switch was used to enable the BI/EMG-triggering of FES when the subject was ready to swallow, which is inconvenient for practical use. In this contribution, a range image camera system is introduced to obtain data of head, mouth, and jaw movements. This data is used to apply a second classification step to reduce the number of false stimulations. In experiments with healthy subjects, the amount of potential false stimulations could be reduced by $47 \%$ while $83 \%$ of swallowing intentions would have been correctely supported by FES.

Key Words: Triggered Functional Electrical Stimulation, Dysphagia, Classification, Range Image Camera, Bioimpedance, Electromyography
\end{abstract}

Eur J Transl Myol 2016; 26 (3):287-291

The EMG and BI signals measured at the larynx show specific patterns during swallowing ${ }^{2}$. The intention to swallow results in an increased activity of the EMG. As soon as the larynx starts to elevate a decent in the bioimpedance can be observed. These patterns can be used to trigger FES in phase to the swallowing intention. ${ }^{1}$ FES is applied over two surface stimulation electrodes which are submentally attached. Stimulation pulses with a frequency of $30 \mathrm{~Hz}$, which have a maximum current amplidude of $25 \mathrm{~mA}$ and a maximum pulse width of $500 \mu \mathrm{s}$, amplify the muscle contraction during swallowing and help the patient to swallow by elavating the larynx and hyoid. The stimulation is applied until the bioimpedance value rises again which indicates the end of the pharyngeal swallowing phase. Unfortunately, swallowing-like patterns in EMG and BI can also be generated by head movements, talking, chewing or food intake and lead to false stimulation triggering. To achieve a more comfortable therapy for dysphagia patients, an improvement of the stimulation triggering is needed. Range image cameras such as the Intel RealSense are capable to detect movements of the head and mouth. The color- and depth image data is used to track parts of the face by calculating landmarks that are placed on designated positions on the face. Fig. 1 shows the positions of the landmarks, that are placed by the Intel RealSense face tracking algorithm, as well as the measurement electrodes for EMG/BI measurement at the neck. We use the camera data to extend the classification process by a second classifier. The flow chart in Fig. 2 illustrates the complete classification process. The EMG/BI signals, that are recorded with the measurement system described in Nahrsteadt et al. ${ }^{3}$, and camera data are synchronized. In a first step, the realtime EMG/BI classifier is used to find all time instances of potential swallow intentions. In a subsequent step we use the camera data to classify in realtime if the EMG/BI pattern was induced by a swallow onset or by a non-swallow related event. Only in the first case, a stimulation is triggered. 


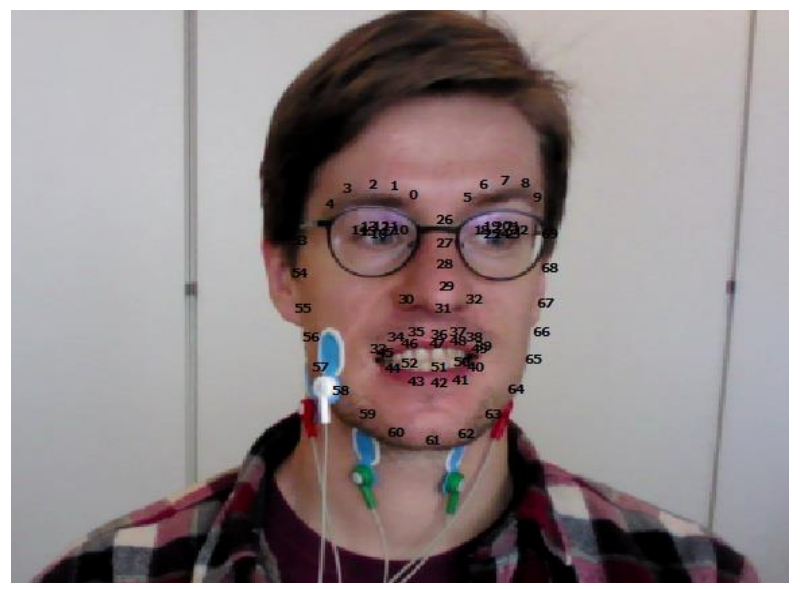

Fig 1. Position of landmarks on the face and measurement electrodes. Courtesy of Benjamin Riebold (first author and subject of the photo)

\section{Materials and Methods}

\section{EMG/BI-based classifier}

The BI signal is downsampled from $2 \mathrm{kHz}$ to $40 \mathrm{~Hz}$ by taking a window of 50 samples, denoising it with a Wavelet algorithm and taking the mean value as downsampled BI value. Based on the work by Nahrstaedt et al. ${ }^{1}$, changes in the downsampled BI signal are detected by a modified sliding window and bottom-up (SWAB) algorithm ${ }^{4}$ which is a piecewise linear approximation method (PLA). The length of the approximated lines is defined by the maximum error maxE between a line and the original data. This concept is enhanced by an upper bound maxL of the line length and a maximum difference $\max D$ between the start and end point of a line. The downsampled BI data are continuously fed into the SWAB algorithm, that creates lines. The parameters of the SWAB algorithm are set to $\max E=1 \Omega, \max D=0.25 \Omega$ and $\max L=1 \mathrm{~s}$. In case a new line is found inside the SWAB algorithm, features are calculated for the EMG/BI classifier. The following features are used:

- SNR of the EMG activity during the newest line,

- last four slope values from the line segments that were found by the SWAB algorithm,

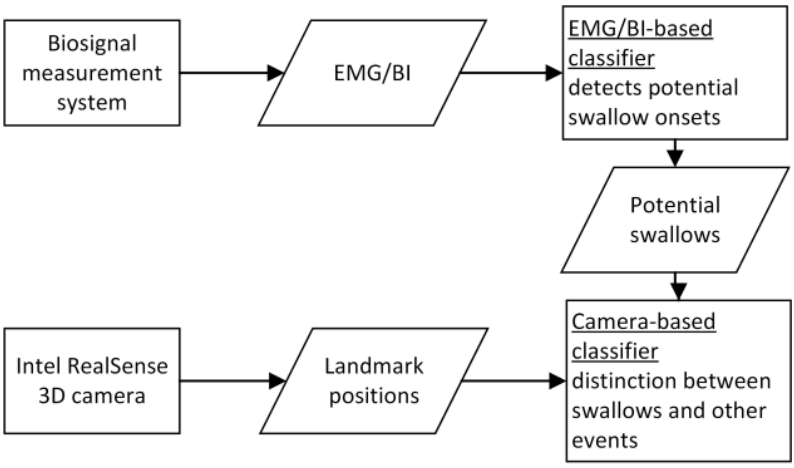

Fig 2. Two-step-classification process.

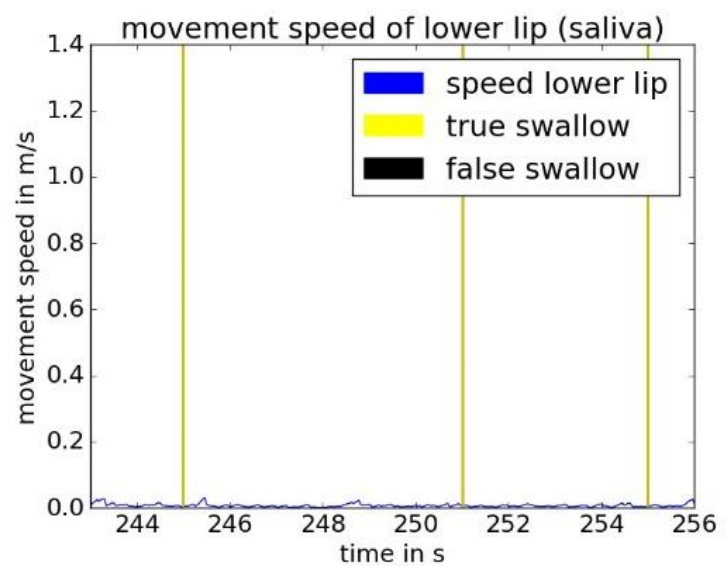

Fig 3. Swallow onsets detected by EMG/BI-based classifier while swallowing saliva.

- $\mathrm{SAX}^{5}$ word from the BI/ EMG data ${ }^{6}$ of the last 0.5 seconds.

The SAX word contains ten symbols and uses an alphabet size of 16 numbers. A random forest classifier is then used to decide which newly created line segment potentionelly belongs to the beginning of a swallow.

\section{Pre-analysis of camera data}

The camera data is used for a second classification of the FES trigger time points generated by the EMG/BI based algorithm. To show how movements result into false classified swallows during the swallowing of saliva and solid boluses, we plotted true and false classified swallows together with the movement speed of the lower lip over time in Fig 3 and Fig. 4, respectively. It can be seen that chewing a solid bolus increases the movement speed of the lips. A higher moving speed is correlated with a higher number of false classified swallows. This can be explained as follows: By moving the jaw down the electrodes get shifted down along the larynx which leads to a drop of BI as well. The activation of the yaw muscles is measured as an EMG activity. Since both effects occur simultaneously, the EMG/BI-based classifier alone would trigger a false stimulation. These events need to be suppressed by the additional camera-based classifier. Movements of the head such as looking up and down or left and right, did not show a strong relation to false stimulation triggering in the data we recorded so far.

\section{Camera-based Classifier}

For a proper classification, significant features have to be extracted from the data in a time window before a potential swallow onset. The first group of features models the movement of the mouth and jaw. For that reason, the moving speed of the upper and lower lip, the jaw and the mouth corners were used as features. We use Eq. 1 to get an approximation of the moving speed of a face part. The landmarks $x_{l}(n)$ contained in a set $L$ 
are differentiated and rectified, summed up and divided by $K$ which is the number of elements in $L$. This is performed for each time instance $n$.

$$
\operatorname{speed}_{L}(n)=\frac{1}{K} \sum_{L}\left|\frac{d x_{l}(n)}{d n}\right|
$$

The definition of the landmarks and the position of the measurement electrodes are shown in Fig. 1.

We used four different sets for feature calculation referring to the upper and lower lip, the mouth corners and the jaw. The sets of landmarks used for feature calculation are summarized in Eq. 2.

$$
\begin{gathered}
L_{\text {upperLib }}=\{35,37,47\} \\
L_{\text {lowerLib }}=\{41,43,51\} \\
L_{\text {mouthCorner }}=\{33,39\} \\
L_{\text {jaw }}=\{61\}
\end{gathered}
$$

A second group of features is used to determine if the mouth is open and if the jaw is deflected downwards. Those features are generated by calculating a distances measure between certain landmarks according to Eq. 3 and Eq. 4.

$$
\begin{aligned}
\operatorname{mouthOpen}(n)= & \frac{\left|x_{43}(n)-x_{35}(n)\right|}{3} \\
& +\frac{\left|x_{47}(n)-x_{51}(n)\right|}{3} \\
& +\frac{\left|x_{37}(n)-x_{41}(n)\right|}{3} \\
\text { jawDown }=\mid & \left|x_{61}(n)-x_{31}(n)\right|
\end{aligned}
$$

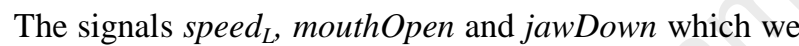
obtain from Eq. 1, Eq. 3 and Eq. 4 are used to calculate the features $f$ according to Eq. 5. That is done by the weighted summation of the values in an interval prior to the potential stimulation trigger time instance $n_{t}$ with a sample length $I$. We used a hanning function $w$ with a width of two times $I$ to weight the samples in the interval. Thus the contribution of a sample to the sum decreases with the distance to $n_{t}$.

$$
f=\sum_{i=0}^{I} w_{2 I}(I-i) \operatorname{Signal}\left(n_{t}-i\right)
$$

The classification of potential trigger points is done using a vector support machine implementation by Chang et al. ${ }^{7}$ We found that radial basis functions as kernel yield the best results. The data was normalized with a standard scaling procedure implemented in scikit-learn. ${ }^{8}$ Thus, each feature was normalized by first subtracting its mean and then dividing by its variance.

To find the best suited classifier, a grid search on the parameters $\gamma$ and $\mathrm{C}$ is performed. The parameter $\mathrm{C}$ weights the influence of the penalty term in the cost function that is used for the vector support machine optimization, whereas $\gamma$ controls the width of the radial basis function used as kernel.

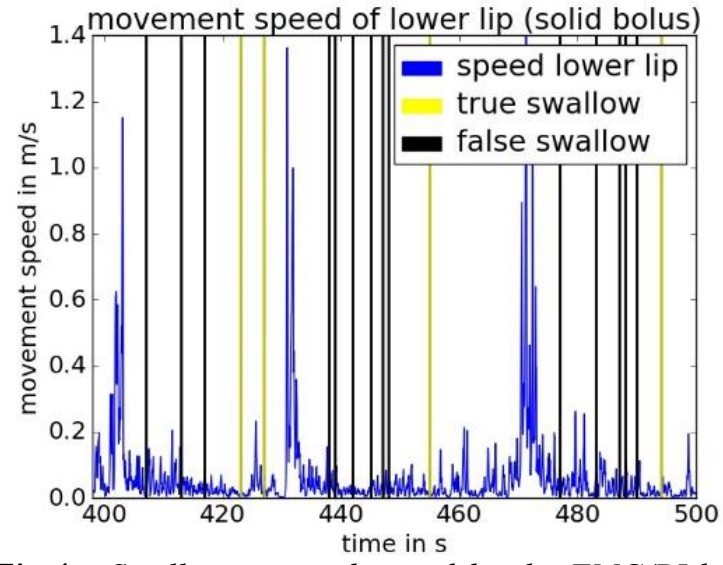

Fig 4. Swallow onsets detected by the EMG/BI-based classifier while swallowing bread.

\section{Evaluation of both classifiers}

The evaluation of the EMG/BI-based and camera-based classifier is performed by using the leave-one- subjectout method by which data from all but one subject are used for training and the remaining data are used for testing. This is repeated for each subject. The safety of the patient is the most important issue. Every swallow intention that is not detected barres the risk of aspiration. For that reason, a suitable classification system needs to have a high sensitivity. The sensitivity $S$ is calculated as shown in Eq. 6. In our case the sensitivity is a measure for the rate of correct classified swallow intentions.

$$
S=\frac{\text { true positives }}{\text { true positives }+ \text { false neagtives }}
$$

\section{Results}

We conducted experiments with five healthy subjects, that were asked to perform three swallows of four different boluses in five different head postures. This study was approved by the ethic board at the Charite Berlin (EA1/161/09). Swallows in the data set have been automatically segmented by using an offline swallow detection ${ }^{6}$. Additionally, the data sets were manually inspecting to confirm or reject found swallows or to mark non-found swallows. In total, 363 swallows are contained in the data set. The resulting data were used as reference for the evaluation of the described two classifiers.

\section{EMG/BI-based classifier}

The best training parameters for the random forest classifier are found to be n_estimators $=40$, min_samples_leaf $=15, \quad$ min_samples_split $=12$ and max_depth=8. A definition of the parameters can be found in the documentation of scikit-learn ${ }^{8}$. The class weights are adjusted inversely to the class frequency by setting the weights option to balanced.

The testing results for all subjects are shown in Table 1. As described by Nahrstaedt et al. $^{6}$, the pharyngeal 
Table 1. BI/EMG-based classfier: Confusion matrix.

\begin{tabular}{lll}
\hline $\begin{array}{l}\text { Potential swallow } \\
\text { onsets }\end{array}$ & $\begin{array}{l}\text { Predicted } \\
\text { positive }\end{array}$ & $\begin{array}{l}\text { Predicted } \\
\text { negative }\end{array}$ \\
\hline True & 335 & 28 \\
False & 273 & 16038 \\
\hline
\end{tabular}

Table 2. Camera-based classifier: Confusion matrix.

\begin{tabular}{lll}
\hline $\begin{array}{l}\text { Potential swallow } \\
\text { onsets }\end{array}$ & $\begin{array}{l}\text { Predicted } \\
\text { positive }\end{array}$ & $\begin{array}{l}\text { predicted } \\
\text { negative }\end{array}$ \\
\hline True & 301 & 34 \\
False & 144 & 129 \\
\hline
\end{tabular}

Table 3. Metrics of camera classification result.

\begin{tabular}{ll} 
Precision & $64 \%$ \\
\hline Pensitivity & $90 \%$ \\
Specificity & $42 \%$ \\
Accuracy & $67 \%$ \\
\hline
\end{tabular}

swallow phase results in a valley in the BI signal with start, minimum and end point. The hyoid bone and larynx have their maximum displacement around the minimum point.

\section{Camera-based classifier}

After applying the grid search over the support vector machine parameters $\gamma$ and $C$ we have chosen the best suited parameter pair for our problem. Among all the classification results we selected the pair with the highest specificity and a sensitivity value above $90 \%(\gamma$ $=0,82 \times 10^{-6}$ and $\left.\mathrm{C}=689.96\right)$. The confusion matrix is shown in Table 2. The corresponding metrics is displayed in Table 3.

In average, swallow onsets were detected $190 \mathrm{~ms}$ after the true begin of a swallow and $369 \mathrm{~ms}$ before the maximal larynx elevation was observed.

\section{Discussion}

The camera-based classifier reduced the number of resulting false stimulations by $47 \%$. However, the number of resulting correct stimulations potential was also sligthy reduced by $11 \%$. The observed timing in the swallow onset detection is sufficient for supporting an elevation of the larynx and hyoid by FES. These preliminary results are a starting point for further investigations. For practical use a higher specificity would be desirable to enable a comfortable therapy. Besides the need of a bigger data set to obtain more generalized trainings data and a more robust evaluation. The swallowing performance of dysphagia patients can be improved by adopting special head postures as shown in different publications. ${ }^{9}$ The postures can be defined by pitch and yaw angles of the head. The desired head posture could be used to switch the stimulation system on and off. This would suppress most of the disturbances and create a more balanced class ratio for the classification system. Those angles can be measured with the Intel RealSense camera and used to give the patients a graphical biofeedback. Combining these two techniques could dramatically increase the stimulation precision.

\section{Contributions}

BR: Data collection and interpretation, development of the camera-based classifier, manuscript writing; HN: Data interpretation, development of the BI/EMG -based classifier, manuscript writing; CS: Study design, manual marking of swallows; ROS: Study Design; TS: Manuscript writing; all: Manuscript Review.

\section{Acknowledgement}

This work was funded by the German Federal Ministry of Economics and Energy (BMWi) within the project MultiEMBI (KF 2392314CS4)

\section{Conflict of Interest}

The authors declare no potential conflict of interests.

\section{Corresponding Author}

Benjamin Riebold, TU Berlin, Sekr. EN 11, Einsteinufer 17, 10587 Berlin, Germany.

E-mail: riebold@control.tu-berlin.de

E-mails of co-authors

Holger Nahrstaedt: nahrstaedt@control.tu-berlin.de Corrina Schultheiss: corinnaschultheiss@gmail.com

Rainer Ottis Seidl: rainer.seidl@ukb.de

Thomas Schauer: schauer@control.tu-berlin.de

\section{References}

1. Nahrstaedt H, Schultheiss C, Schauer T, Seidl RO. Bioimpedance- and EMG-Triggered FES for Improved Protection of the Airway During Swallowing. Biomedical Engineering / Biomedizinische Technik 2013.

2. Schultheiss C, Schauer T, Nahrstaedt H, Seidl RO. Automated Detection and Evaluation of Swallowing Using a Combined EMG/Bioimpedance Measurement System. The Scientific World Journal 2014;2014:405471.

3. Nahrstaedt H, Schauer T, Seidl RO. Bioimpedance based measurement system for a controlled swallowing neuro-prosthesis. Proc. of 15th Annual International FES Society Conference and 10th Vienna Int Workshop on FES 2010:49-51.

4. Keogh E, Chu S, Hart D, Pazzani M. An online algorithm for segmenting time series. Proceedings 2001 IEEE International Conference on Data Mining: IEEE; 2001:289-96.

5. Lin J, Keogh E, Lonardi S, Chiu B. A symbolic representation of time series, with implications for streaming algorithms. Proceedings of the 8th ACM 


\section{Triggering FES to Support Voluntary Swallowing}

Eur J Transl Myol 26 (4): 287-291

SIGMOD workshop on Research issues in data mining and knowledge discovery: ACM 2003:2.

6. Nahrstaedt H, Schultheiss C, Seidl RO, Schauer T. Swallow Detection Algorithm Based on Bioimpedance and EMG Measurements. 8th IFAC Symposium on Biological and Medical Systems. Budapest, Hungary; 2012:91-6.

7. Chang C-C, Lin C-J. LIBSVM: A library for support vector machines. ACM Trans. Intell. Syst. Technol. 2011;2:1-27.
8. Pedregosa F, Varoquaux G, Gramfort A, et al. Scikit-learn: Machine Learning in Python. Journal of Machine Learning Research 2011;12:2825-30.

9. Macrae P, Anderson C, Humbert I. Mechanisms of Airway Protection During Chin-Down Swallowing. J Speech Lang Hear Res 2014;57:1251 